\title{
Histopathological Findings in Symptomatizing Patients After Supracervical Hysterectomy: A Cross Sectional Study
}

\author{
Ayman S Dawood ${ }^{1}$, Heba Harras ${ }^{2 *}$ and Hashem A Lotfy ${ }^{1}$ \\ ${ }^{1}$ Department of Obstetrics and Gynecology, Tanta University, Egypt \\ ${ }^{2}$ Department of Pathology, Tanta University, Egypt
}

*Corresponding author: Heba Fouad Harras, Lecturer of pathology, Faculty of medicine, Tanta University Tanta, Egypt.

Received Date: March 18, 2021

Published Date: May 28, 2021

\begin{abstract}
Background: Supra-cervical hysterectomy ( $\mathrm{SCH}$ ) is widely common and has many complications either immediate or delayed, including bleeding, infection, and chronic pelvic pain. Clinical studies evaluating histopathological findings in these patients are few.

Objective: To study the underlying pathological changes in the cervical stump after supracervical hysterectomy in symptomatizing patients.

Patients and Methods: This cross-section study was conducted at Tanta University. All patients $(\mathrm{n}=132)$ underwent cervical stump biopsy for histopathological examinations. Immunohistochemical expression of P16 was also performed in all patients with cervical pathological abnormalities
\end{abstract} as a recommended biomarker for cervical lesions.

Results: Vaginal bleeding was the most common presentation of enrolled patients (54/132). Endometriosis was the commonest pathological lesion detected in patients with cervical stump bleeding (27/54). After Hematoxylin and eosin staining was applied; 52 cases showed normal cervical tissue, chronic non-specific cervicitis in 11 cases, endometriosis in 27 cases, squamous metaplasia with no atypia in 6 cases, cervical intraepithelial neoplasia in 32 cases, squamous cell carcinoma in 3 cases and adenocarcinoma in only one case. P16 immuno-staining showed negative expression in chronic cervicitis and squamous metaplasia with no atypia, ambiguous p16 expression was observed in 50\%, $63.2 \%$ and $80 \%$ cases of CIN I, CIN II, and III respectively, while $100 \%$ of cervical cancer cases showed block positive expression.

Conclusion: Pathological lesions of cervical stump following SCH could be screened by p16 immuno-staining as a complementary test for early detection of cervical cancer. Ambiguous expression of p16 should not be neglected as the lesion may have a low possibility of harboring high-risk human papilloma.

Keywords: Cervical stump; Histopathology; p16 expression; Supracervical hysterectomy

Abbreviations: BMI: Body Mass Index; CIN: Cervical Intraepithelial Neoplasia; H\&E: Hematoxylin and Eosin; HSIL: High-grade Squamous Intraepithelial Lesions; HPV: Human Papilloma Virus; IHC: Immunohistochemistry; SCH: Supracervical Hysterectomy.

\section{Introduction}

Hysterectomy is the second most common major operation after cesarean section, that met within gynecological and obstetrics clinic. By the age of 65 , more than a third of all women are expected to have had this surgery [1].
In subtotal supracervical hysterectomy, only the body of the uterus is removed compared to the total hysterectomy where the cervix along with the uterus are excised. In the last century, it was thought that leaving the cervix in place preserve better bowel, urinary and 
sexual function as well as avoidance of post- total hysterectomy complications as vaginal cuff abscesses, urethral injury, and incontinence. A meta- analysis of nine randomized studies of women with a history of supracervical or complete hysterectomy for benign gynecologic disorders disapproved this claim. There was no difference in sexual function or incontinence after surgery [2]. However, cervical stump symptoms can cause some sort of distress in certain individuals to require further operation and cervical stump excision [3].

The current studies on risk factors for constant postoperative cervical stump bleeding are inconclusive. Some research found endometriosis to be a risk factor [4], whereas others failed to find such a link [3]. The data on endocervical removal during hysterectomy were inconclusive [5]. The main disadvantage of subtotal hysterectomy is still the risk of developing cervical stump cancer and the need for regular cervical screening following the surgery [6].

Although the screening programs using pap smears are highly successful and being used routinely in some countries, a significant number of cases of cervical cancer have still been missed which can be attributed to false negative test results due to sampling errors, inter and intra observer variability [7].

The diagnostic interpretation of hematoxylin and eosin (H\&E)stained cervical biopsies is subject to substantial variability between readers, leading to potential under-treatment of women with high-grade precancerous lesions (high-grade squamous intraepithelial lesions [HSIL]) or greater, or overtreatment in case of false-positive diagnostic interpretations $[8,9]$.

This makes a bad need for a more sensitive and specific test for improving cervical cancer screening and accurately diagnosing precancerous lesions [10]. Since the lower anogenital terminology (LAST) project performed by the American Society for Colposcopy and cervical pathology(ASCCP) and the college of American pathology(CAP), the published literature recommended the use of biomarkers to improve diagnostic agreement [11]. It has been suggested that p16 staining is a recommended biomarker for cervical lesions [12]. However, even today the use of p16 protein as a prognostic biomarker of cervical cancer remains controversial.

In the view of the current study, symptomatizing patients after SCH was recruited and biopsy was taken to show what are the underlying pathological findings of the cervical stump in those patients, and to aid for putting the comprehensive therapeutic strategy for their treatment. The study also was designed to evaluate the immuno-staining pattern of p16 compared to histopathological diagnosis and its accuracy in diagnosis and interpretation of cervical biopsy results.

\section{Patients and Methods}

\section{Study design and settings}

This study is a cross section descriptive study conducted at Tanta University Hospital, in both Obstetrics \& gynecology and Pa- thology departments. The study was conducted in the period from December 2017 till January 2021.

\section{Patients}

All symptomatic patients following hysterectomy were recruited and eligible patients were included according to inclusion and exclusion criteria. The inclusion criteria were (a) Any age (b) Supracervical hysterectomy, (c) Symptomatic patients. The exclusion criteria were (a) Total hysterectomy, (b) hysterectomies for malignant lesions indications, and (c) Patients refusing to participate.

\section{Sample size calculation}

Sample was calculated using Epi-Info 2000 statistical program. The standard normal variate (at 5\% type 1 error) was 1.96, the expected prevalence of cervical cancer in stump up to $9 \%$ [13], p-value of 0.05 , the sample was 110 cases.

\section{Methods}

All patients' demographic data, indication of hysterectomy, duration since operation, postoperative complications, latent period, and their main complaint were recorded.

\section{Interventions}

Gynecological interventions: Under general anesthesia, cervical biopsy (4-quadrant punch biopsy) was taken from all patients, put in formalin sterile container, and sent for histopathological examination.

Histopathological examination: All the received cervical specimens were formalin fixed and paraffin embedded. Hematoxylin and eosin (H\&E)-stained slides were subjected to routine histopathological examination. The pathological findings were categorized into; endometriosis, non-specific cervicitis, squamous metaplasia, cervical intraepithelial neoplasia (CIN) and cancer cervix. Cervical intraepithelial neoplasia is classified into; CIN I, CIN II, and CIN III according to how much tissue affected by dysplasia; lower one third, lower two thirds or more than two thirds respectively [14].

\section{P16 immunohistochemistry and interpretation}

After exclusion of cases showed normal and endometriosis histological finding, 53 cervical biopsy specimens were subjected to IHC staining using p16 mouse monoclonal antibody (5A8A4) catalogue $\neq$ MA5-17093 (ThermoFisher scientific) according to the standard protocol. After deparaffinizing $4 \mu \mathrm{m}$ thickness tissue sections and rehydration in graded alcohols, antigen retrieval was carried out in a pressure cooker using an ethylene diamine tetra acetic acid buffer. The process used was horseradish peroxidase. 100 microliters of the primary antibody were added to each slide. After the enhancer, the secondary antibody was added. The chromogen was diaminobenzidine, and the substrate was $\mathrm{H}_{2} \mathrm{O}_{2}$. The slides were counterstained with hematoxylin and dehydrated using grades of alcohol. They were then cover slipped with DPX after being cleared in xylene. 
Immunohistochemical (IHC) expression for p16 in the nucleus is regarded positive. The pathologist went over all of the IHC slides and wrote down a detailed description of p16 immunostaining in each lesion based on 4 criteria: (1) intensity: strong versus weak ; (2) extent: diffuse versus focal; (3) continuity: continuous versus discontinuous; and (4) position of positive cells (in the lowest third, two thirds, or complete epithelial thickness. Based on these criteria, lesions were classified as block-positive, negative, and ambiguous pattern. Block-positive patterns met all LAST requirements for being strong and diffuse. The absence of staining was categorized as negative expression. Certain p16 results that fit some but not all requirements for the "block-positive" pattern were classed as ambiguous which were furtherly identified in three patterns: (1) strong/ basal (2) weak/diffuse and (3) strong/ focal with discontinuous staining located at any level of the epithelium) [15].

\section{Ethical approval and study registration}

This study was approved from the local ethical committee of Tanta University before the start of enrollment and given the following code $31895 / 11 / 17$. Also, this study was registered at clinicaltrials.gov and given the following ID: NCT04809727 and is available on the following link: https:/ /register .clinicaltrials .gov/prs /app/ action/SelectProtocol?sid=S000ASC0\&selectaction=Edit\&uid=U$000404 \mathrm{~W} \& \mathrm{ts}=2 \& \mathrm{cx}=-\mathrm{rdza} 4 \mathrm{~s}$

Table 1: Demographic data of enrolled patients $(n=132)$.

\begin{tabular}{|c|c|c|}
\hline Demographic data & Range & $($ mean \pm SD) \\
\hline Age (in years) & $37-63$ & $51.40 \pm 6.44$ \\
\hline Gravidity & $0-9$ & $3.1 \pm 1.3$ \\
\hline Parity & $0-5$ & $2.6 \pm 0.98$ \\
\hline BMI & $22-41$ & $31.85 \pm 4.70$ \\
\hline Duration since hysterectomy (years) & $1-\quad 10$ & $4.21 \pm 1.86$ \\
\hline \multirow[t]{2}{*}{ Interval (years) } & $0.5-6$ & $3.13 \pm 1.09$ \\
\hline & Number & Percentage \\
\hline Follow up following hysterectomy & 5 & 3.8 \\
\hline \multicolumn{3}{|l|}{ Route of hysterectomy } \\
\hline Abdominal & 104 & 78.8 \\
\hline Laparoscopic & 28 & 21.2 \\
\hline \multicolumn{3}{|l|}{ Residence } \\
\hline Urban & 75 & $56.80 \%$ \\
\hline Rural & 57 & $43.20 \%$ \\
\hline \multicolumn{3}{|l|}{ Associated diseases } \\
\hline DM & 27 & $20.50 \%$ \\
\hline Hypertension & 27 & $20.50 \%$ \\
\hline DM and Hypertension & 6 & $4.50 \%$ \\
\hline NO & 72 & $54.50 \%$ \\
\hline
\end{tabular}

BMI: body mass index

DM: diabetes mellitus

Citation: Ayman S Dawood, Heba Harras, Hashem A Lotfy. Histopathological Findings in Symptomatizing Patients After Supracervica Hysterectomy: A Cross Sectional Study. W J Gynecol Women's Health. 5(1): 2021. WJGWH.MS.ID.000601.

DOI: 10.33552/WJGWH.2021.05.000601.

\section{Statistical methods}

The full detailed form is SPSS 20, IBM, Armonk, NY, United States of America. Quantitative data were expressed as mean \pm standard deviation (SD). Qualitative data were expressed as frequency and percentage. Independent-samples t-test of significance was used when comparing between two means.

\section{Results}

\section{Demographic data of the enrolled patients (Table 1)}

The patient' s mean age in this study was $51.40 \pm 6.44$ years, range $37-63$ years. The mean gravidity was $3.1 \pm 1.3$, ranged from $0-9$ and the mean parity was $2.6 \pm 0.98$, ranged from 0 - 5 . The mean BMI was $31.85 \pm 4.70$ with range of $22-41$. The mean duration since hysterectomy was $4.21 \pm 1.86$ years. Interval (latent period) was ranged from $0.5-6$ with mean of $3.13 \pm 1.09$. Most of patients were operated with abdominal open approach $(78.8 \%)$ while $21.2 \%$ of cases were operated with laparoscopic approach. Follow up following hysterectomy was found to be in 5 cases (3.8\%) only in the form of pap smear. Regarding the residence, seventy-five cases (56.8\%) were urban and 57 (43.2\%) lived in rural areas. Out of 132 studied cases; 27 (20.5\%) cases were diabetics, 27 (20.5\%) were hypertensive, 6 cases ( $4.5 \%)$ were diabetic and hypertensive. The remaining $72(54.5 \%)$ cases have no history of associated diseases. 


\section{Reasons for having supracervical hysterectomy (Figure} 1)

The main indication for hysterectomy was uterine fibroids in $41(31.1 \%)$ cases, followed by dysfunctional uterine bleeding in 30
(22.7\%), endometrial hyperplasia in 15 (11.4\%), peripartum hemorrhage in 13 (9.8\%) and endometriosis in 17 (12.9\%). Other reasons were noticed; chronic pelvic pain and genital prolapse in 12 $(9.1 \%)$ and $4(3 \%)$ respectively.

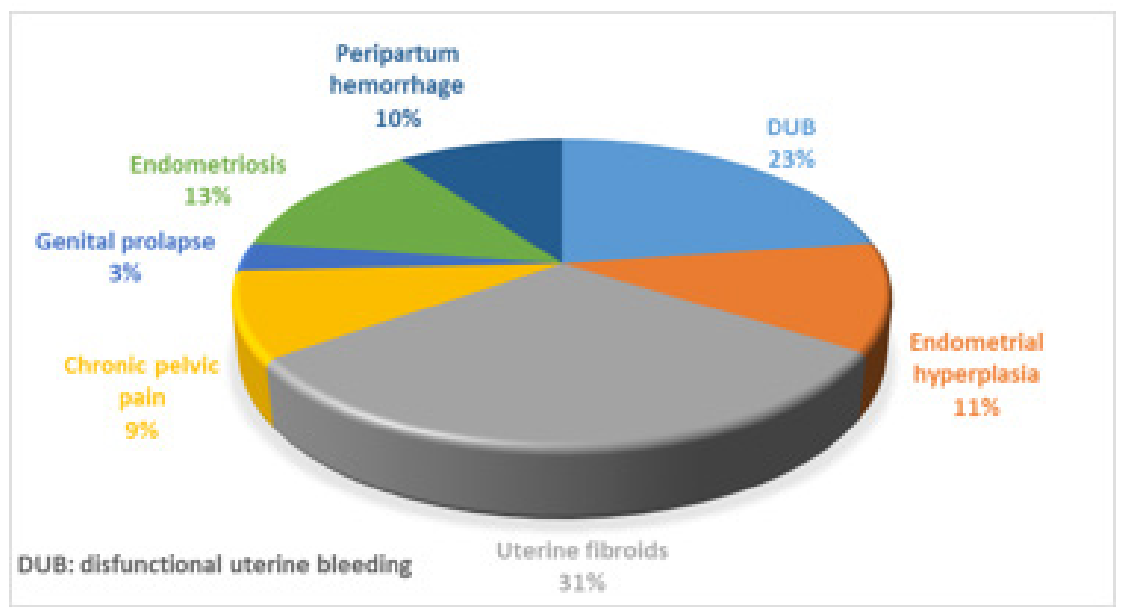

Figure 1: Indications of supracervical hysterectomy $(\mathrm{N}=132)$.

\section{Post-supracervical hysterectomy symptoms (Table 2)}

Among 132 studied cases who reported symptoms related to the cervical stump, abnormal bleeding was present in 54 (40.9\%) cases. Twenty-five (18.9\%) women suffered from chronic pelvic pain. Twenty-two (16.7\%) and 15 (11.4\%) women asked for consultation due to excessive discharge and dry vagina, respectively. Dyspareunia was reported in 9 (6.8\%) cases and urinary symptoms in $7(5.3 \%)$ cases.

Table 2: Clinical post- supracervical hysterectomy presentations of enrolled patients $(\mathrm{N}=132)$.

\begin{tabular}{|c|c|}
\hline Presentation & N (\%) \\
\hline Abnormal bleeding & $54(40.9 \%)$ \\
\hline Chronic pelvic pain & $25(18.9 \%)$ \\
\hline Excessive discharge & $22(16.7 \%)$ \\
\hline Dry vagina & $15(11.4 \%)$ \\
\hline Dyspareunia & $9(6.8 \%)$ \\
\hline Urinary symptoms & $7(5.3 \%)$ \\
\hline
\end{tabular}

The mean age of patients suffering from post-supracervical hysterectomy bleeding was significantly lower than those with other symptoms $(\mathrm{p}<0.05)$ while the mean of body mass index $(B M I)$ was higher in patients with bleeding compared to those with other symptoms (Table 3).

Table 3: Correlation between post supracervical hysterectomy bleeding and both age and BMI.

\begin{tabular}{|c|c|c|c|c|c|}
\hline & & Abnormal Bleeding & Other symptoms & T. test & P. value \\
\hline \multirow{2}{*}{$\begin{array}{c}\text { Age } \\
\text { (in years) }\end{array}$} & Range & $39-54$ & $37-63$ & \multirow[t]{2}{*}{3.33} & \multirow[t]{2}{*}{$0.001^{*}$} \\
\hline & Mean \pm SD & $46.2 \pm 4.27$ & $49.54 \pm 6.45$ & & \\
\hline \multirow[t]{2}{*}{ BMI } & Range & $21-41$ & $23-41$ & \multirow[t]{2}{*}{3.23} & \multirow[t]{2}{*}{$0.002^{*}$} \\
\hline & Mean \pm SD & $33.37 \pm 4.39$ & $30.77 \pm 4.64$ & & \\
\hline
\end{tabular}

*significant $<0.05$

BMI: Body Mass Index 


\section{Histopathological findings (Table 4 \& Figure 2)}

Of 132 studied cases; 52 (39.4\%) cases showed normal cervical tissue. Chronic non-specific cervicitis was found in 11 (8.3\%) (Figure 2A), endometriosis (Figure 2B) in 27 (20.5\%), squamous metaplasia with no atypia (Figure 2C) in 6 (4.5\%) and cervical intraepithelial neoplasia in $32(24.2 \%)$ cases; 8 cases were CINI (Figure 2D), 19 cases were CINII (Figure 2E) and 5 cases were CIN III (Figure 2F). Three cases (2.3\%) were poorly differentiated invasive squamous cell carcinoma (Figure $2 \mathrm{G}$ ) and only one case of Villoglandular adenocarcinoma (0.75\%) (Figure $2 \mathrm{H})$.

Table 4: Histopathological cervical biopsy finding in symptomatizing post supra-cervical hysterectomy studied cases ( $n=132)$.

\begin{tabular}{|c|c|c|}
\hline \multicolumn{2}{|c|}{ Histopathological Diagnosis } & Number of cases (\%) \\
\hline \multicolumn{2}{|c|}{ Normal cervix } & $52(39.4 \%)$ \\
\hline \multicolumn{2}{|c|}{ Chronic nonspecific cervicitis } & $11(8.3 \%)$ \\
\hline \multicolumn{2}{|c|}{ Endometriosis } & $27(20.5 \%)$ \\
\hline \multicolumn{2}{|c|}{ Squamous metaplasia } & $6(4.5 \%)$ \\
\hline \multirow{3}{*}{$\begin{array}{c}\text { CIN } \\
32(24.2 \%)\end{array}$} & CIN I & $8(6.06 \%)$ \\
\hline & CIN II & $19(14.4 \%)$ \\
\hline & CIN III & $5(3.8 \%)$ \\
\hline \multicolumn{2}{|c|}{ Squamous cell carcinoma } & $3(2.3 \%)$ \\
\hline \multicolumn{2}{|c|}{ Adenocarcinoma } & $1(0.75 \%)$ \\
\hline
\end{tabular}

CIN: Cervical Intraepithelial Neoplasia

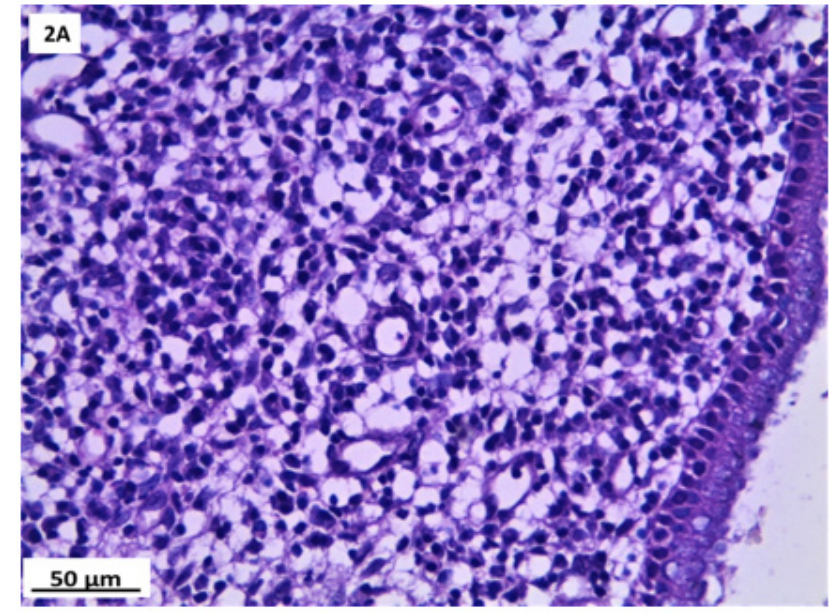

Figure 2A: Chronic nonspecific cervicitis: severe inflammatory cell infiltrate with intercellular oedema (x400).

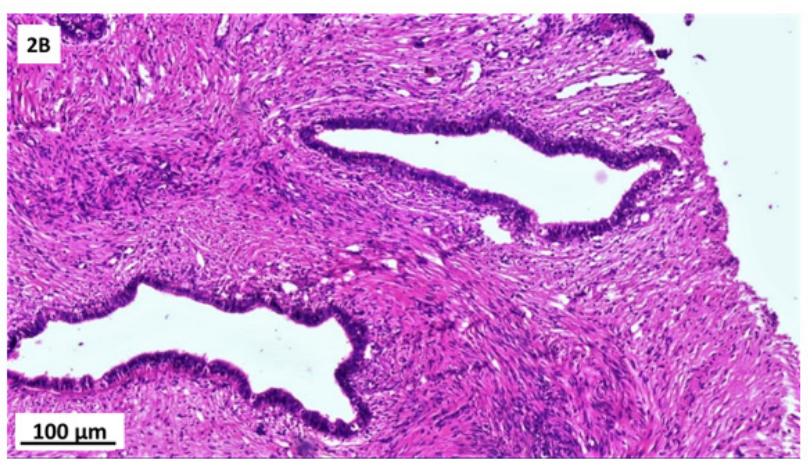

Figure 2B: Endometriosis of cervix: endometrial glands and stroma are embedded within the cervical tissue (x200). 


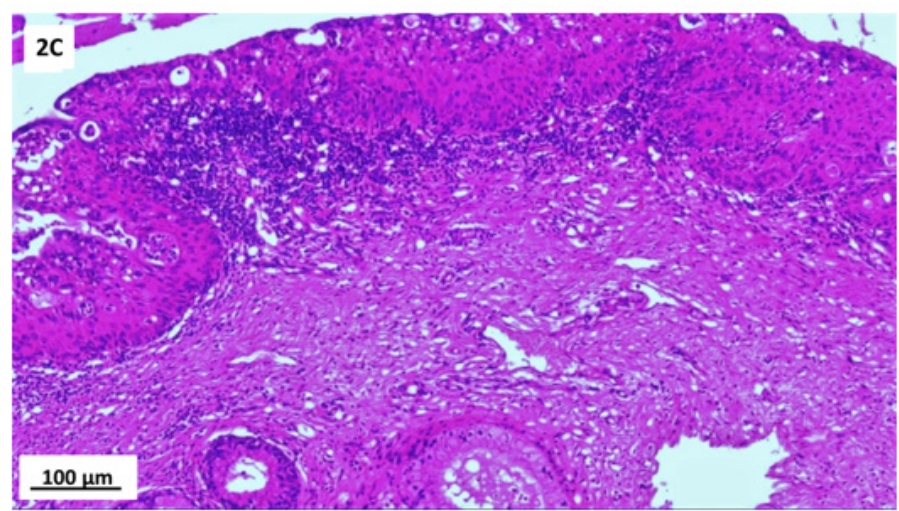

Figure 2C: Squamous metaplasia: replacement of surface columnar epithelium by non-keratinized squamous epithelium without atypia composed of polygonal cells with abundant eosinophilic cytoplasm (x200).

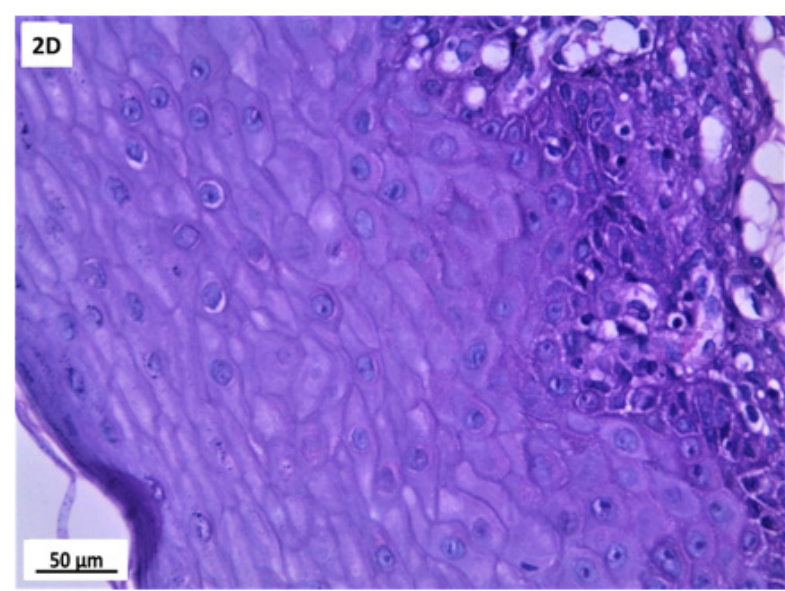

Figure 2D: CINI: dysplastic cells with nuclear angulation, located at the lower third of the epithelium (x400).

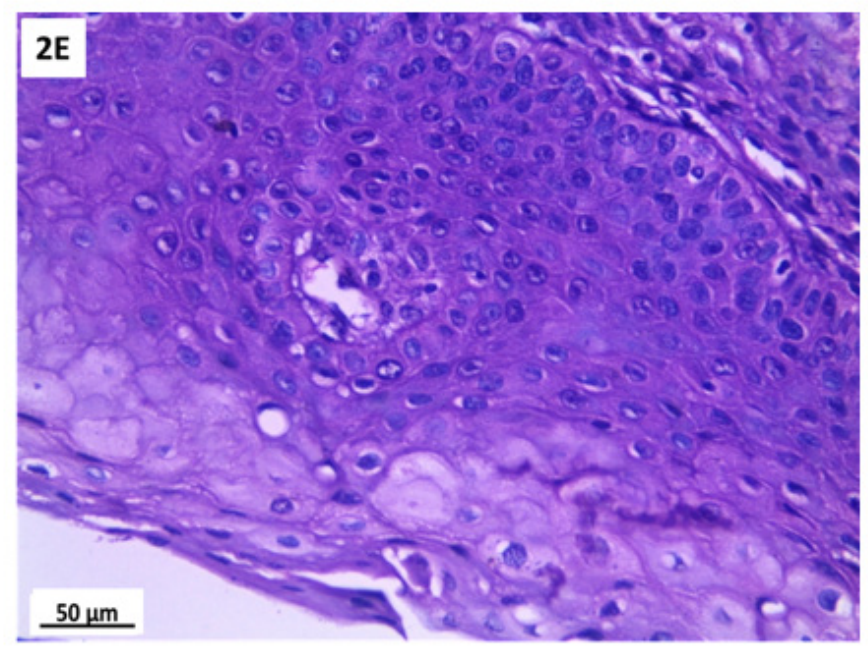

Figure 2E: CINII: dysplastic cells are in the lower two thirds of the surface epithelium (x400). 


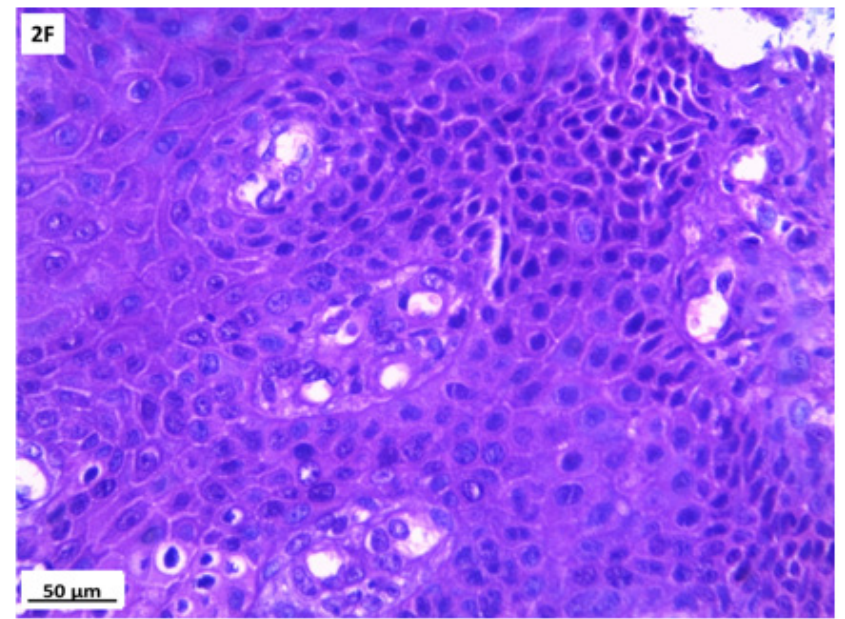

Figure 2F: CINIII: dysplastic cells span more than the lower two thirds of surface epithelium (x400).

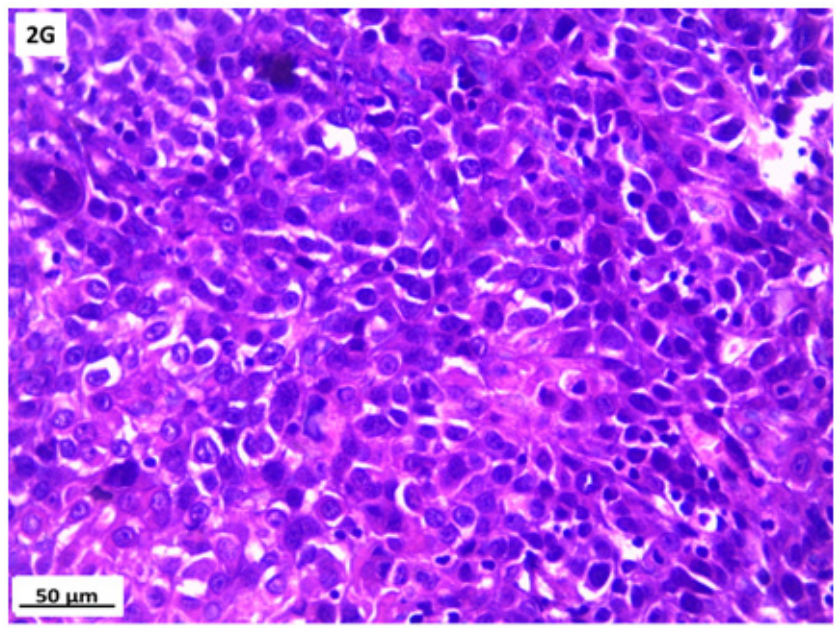

Figure 2G: Poorly differentiated squamous cell carcinoma: infiltrating nests and sheets of non-keratinizing malignant large cells with moderate amount of amphophilic cytoplasm and prominent nucleoli (x400).

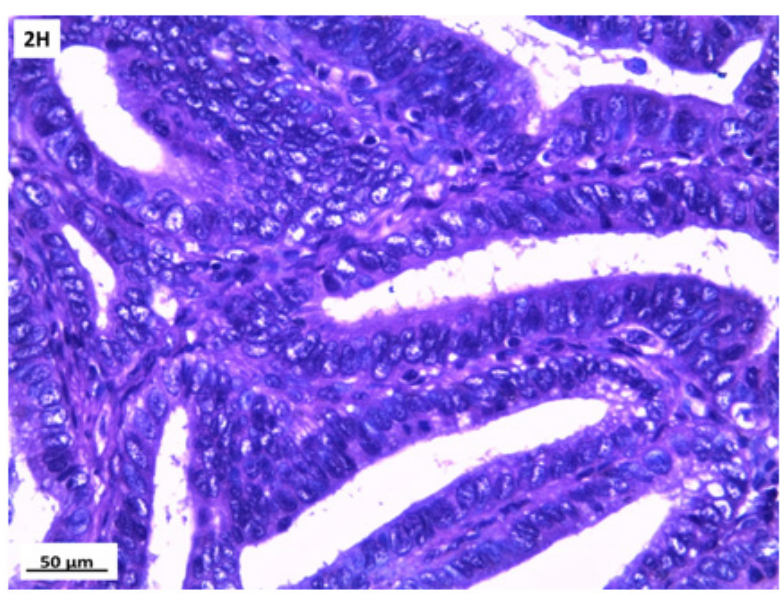

Figure $2 \mathrm{H}$ : Villoglandular papillary adenocarcinoma: exophytic growth with long slender papillae lined by several layers of malignant cells $(\mathrm{x} 400)$. 
Among 54 patients complaining of post supracervical hysterectomy bleeding; 27 cases showed endometriosis, 4 cases showed squamous metaplasia, 19 cases showed cervical intraepithelial neoplasia and 4 cases showed cervical carcinoma.

\section{P16 immunostaining expression (Table 5, Figure 3)}

P16 expression was evaluated in 53 cases after exclusion of cases with normal cervix and endometriosis. All the inflammatory and squamous metaplasia specimens showed no expression of p16, while ambiguous p16 immunostaining was detected in 4/8 of CIN I (Figure 3A), 12/19 of CIN II (Figure 3B,3C) and 4/ 5 of CIN III. All the invasive squamous cell carcinoma 3/3 (Figure 3D) and adenocarcinoma1/1 (Figure 3E) cases showed diffuse strong positive expression.

Table 5: $\mathrm{p} 16$ expression in cervical biopsy with various histopathological findings ( $N=53$ ).

\begin{tabular}{|c|c|c|c|c|}
\hline \multirow{2}{*}{ Histopathological Diagnosis } & Number of cases (\%) & \multicolumn{3}{|c|}{ P16 expression } \\
\cline { 3 - 4 } & & Positive & Ambiguous & Negative \\
& & $\mathrm{N}(\%)$ & $\mathrm{N}(\%)$ & $0(\%)$ \\
\hline Non-Specific cervicitis & $11(100)$ & $0(0)$ & $0(0)$ & $6(11(100)$ \\
\hline Squamous Metaplasia & $6(100)$ & $0(0)$ & $4(50)$ & $4(50)$ \\
\hline CIN I & $8(100)$ & $0(0)$ & $12(63.2)$ & $7(36.8)$ \\
\hline CIN II & $19(100)$ & $0(0)$ & $4(80)$ & $1(20)$ \\
\hline CIN III & $5(100)$ & $3(100)$ & $0(0)$ & $0(0)$ \\
\hline Squamous cell carcinoma & $3(100)$ & $1(100)$ & $0(0)$ & $0(0)$ \\
\hline Adenocarcinoma & $1(100)$ & $4(7.6)$ & $20(37.7)$ & $29(54.7)$ \\
\hline Total & $53(100)$ & & \\
\hline
\end{tabular}

CIN: Cervical Intraepithelial Neoplasia

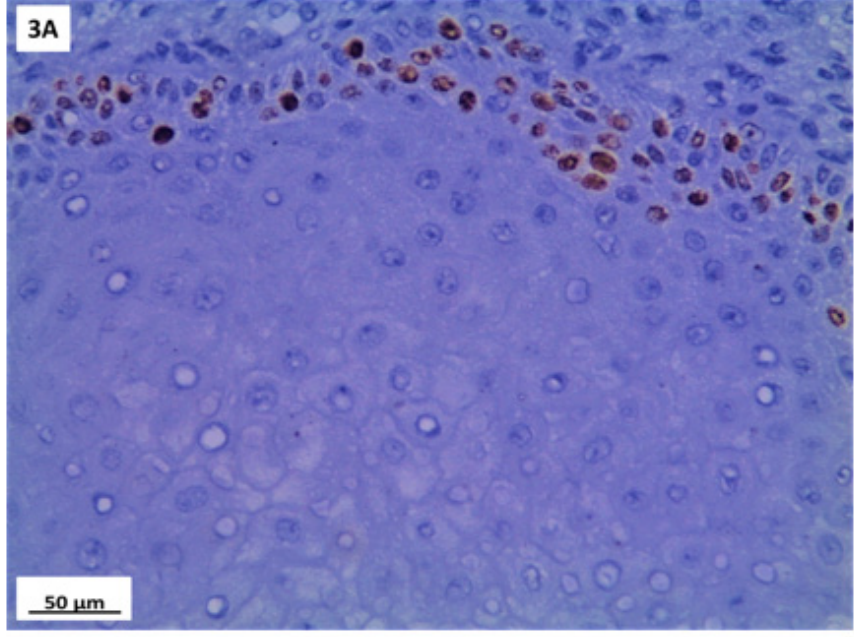

Figure 3A: CINI showing p16 ambiguous (strong basal) expression (x400). 


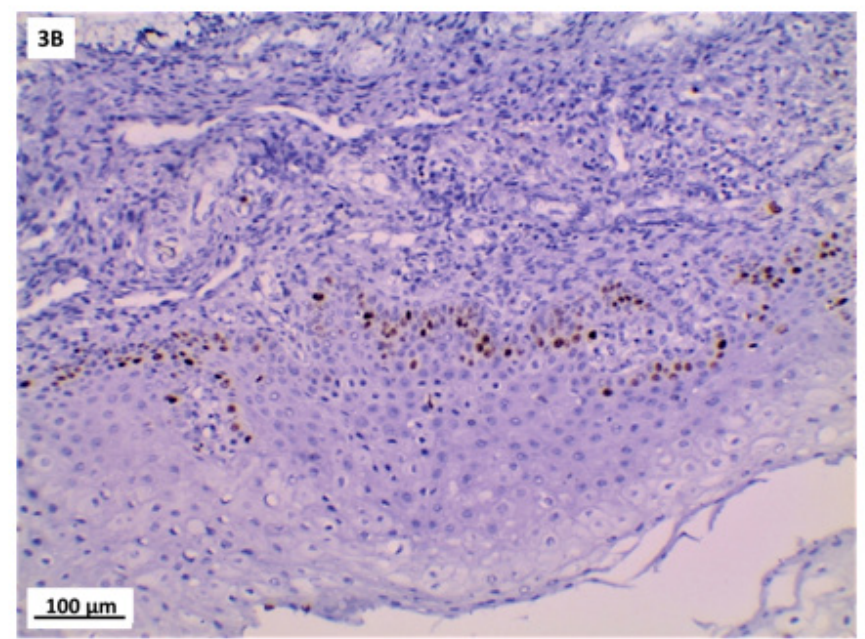

Figure 3B: CINII showing p16 ambiguous (strong basal) expression (x200).

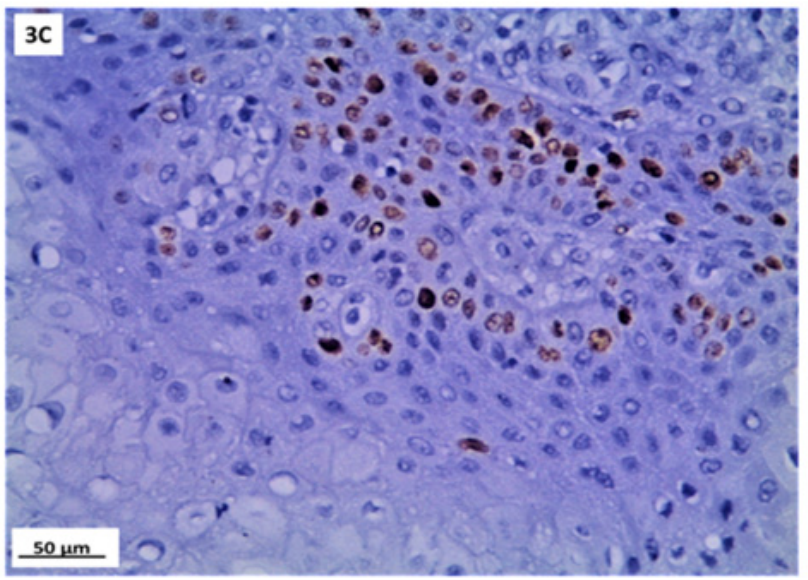

Figure 3C: Another case of CINII showing p16 ambiguous (strong focal) expression (x400).

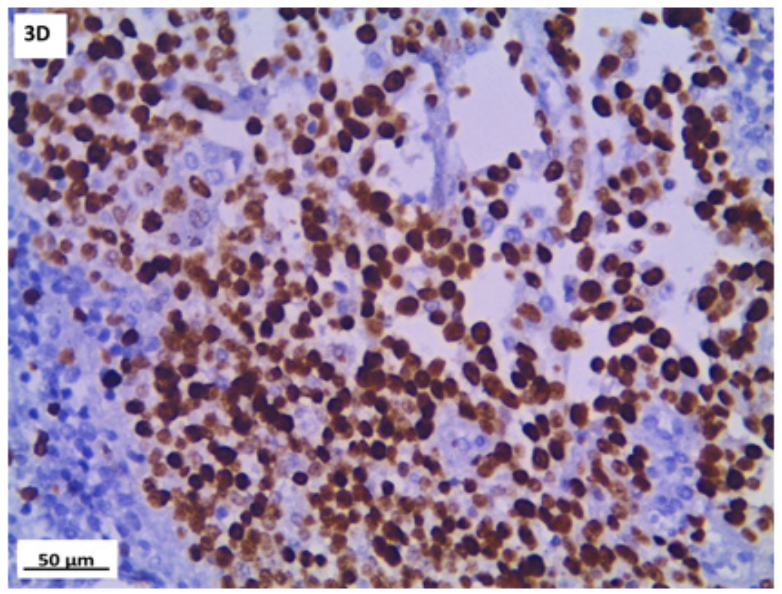

Figure 3D: Squamous cell carcinoma showing p16 block positive (diffuse strong) expression ( $x 400)$. 


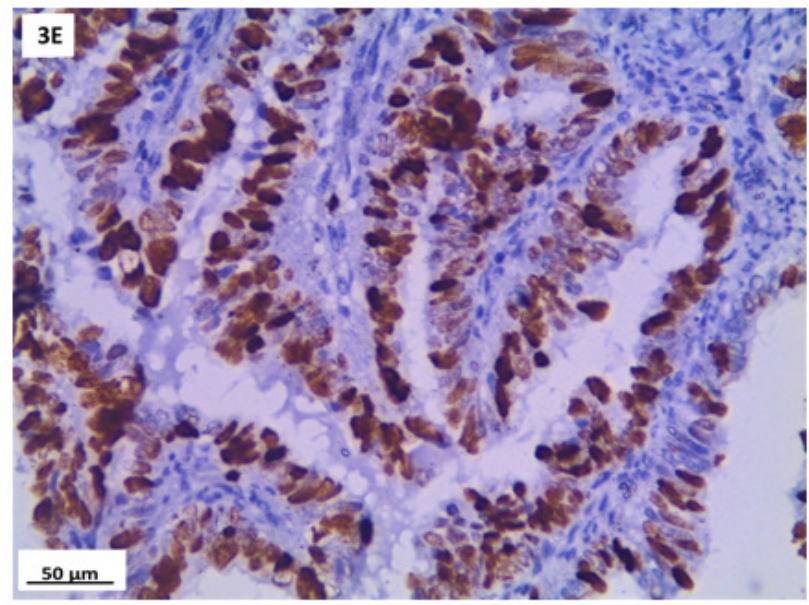

Figure 3E: Adenocarcinoma showing p16 block positive (diffuse strong) expression (x400).

\section{Discussion}

Hysterectomy can be done either through abdominal or laparoscopic approach. In our study, 104 hysterectomy cases $(78.8 \%)$ were performed abdominally and the remaining 28 (21.2\%) were carried out laparoscopically. Dawood, et al. [16] conducted a gynecologists' survey study in Egypt and reported that abdominal route was the most common route for hysterectomy with subtotal type accounting for $71.5 \%$ which is in agreement with our results.

This study reports the occurrence of cervical stump symptoms after supracervical hysterectomy (SCH). The main complaint was bleeding followed by chronic pelvic pain then excessive discharge, dry vagina, dyspareunia, and urinary symptom.

The most common complication was vaginal bleeding (40.9\%). Several previous studies reported its occurrence after SCH to be in the wide range of $0-25 \%[4,5,17]$. In our study, it was relatively high compared to these previous reports and was found to be higher in young ages than those with other cervical stump symptoms. Elbohoty, et al. [18] conducted a survey study to evaluate the percentage of symptomatic patients following supracervical hysterectomy and they found that $32.31 \%$ of patients were symptomatic. They reported that offensive vaginal discharge was the commonest presentation 15 (11.54\%), followed by abnormal vaginal bleeding 11 (8.46\%), chronic pelvic pain 5 (3.85\%) and sexual dysfunction 5 (3.85\%). The lower percentages of presentations are due to inclusion of non-symptomatic patients in their study.

Body mass index (BMI), as a risk factor for postoperative bleeding, is a variable that has received little attention in the literature. In the present study, it was significantly higher in patients with bleeding than those with other post SCH symptoms. This finding is in accordance with Sasaki, et al. [19] who detected positive significant correlation between post-operative vaginal bleeding and BMI and explained it by the fact that a higher BMI often indicates greater adi- posity, which implies increased peripheral conversion of androgens to estrogen and possibly increased persistent bleeding caused by hormonal stimulation of any remaining endometrium.

In the present study, out of twelve women who reported suffering from preoperative pelvic pain, five patients showed persistent pelvic pain after SCH. This more or less agrees with Lieng, et al. [6] who observed continued periodic pain in $21 \%$ of cases following their hysterectomy.

Histopathological analysis in symptomatizing post SCH patients in our study showed normal cervical tissue in 39.4\% and cervical intraepithelial neoplasia ( $\mathrm{CIN}$ ) in $24.2 \%$ which is the most prevalent pathological finding, followed by endometriosis in $20.5 \%$, chronic nonspecific cervicitis in $8.3 \%$, squamous metaplasia in $4.5 \%$ and the least common one was cancer cervix in $3.03 \%$; Squamous cell carcinoma in $2.3 \%$ and adenocarcinoma in $0.75 \%$.

In our study, seventeen cases were suffering from endometriosis as an indication for hysterectomy, ten of them showed endometriosis of the cervical stump. Okaro, et al. [4] who reported endometriosis in $23.5 \%$ of cases, found Fourteen out 17 studied cases with cervical stump symptoms had been previously treated for endometriosis, and identified endometriosis as a substantial risk factor for cervical stump symptoms, which could make this surgery contraindicated. They also suggested that the original operative technique may fail to remove all the uterine corpus and endocervical canal in these patients. Sasaki, et al. [19] explained bleeding in these cases as patients continued to produce estrogen, the ectopic endometrial tissue stimulated postoperative vaginal bleeding. Several studies suggested that endometriosis may have arisen de novo or have been residual deposits [4].

In the present study we found that endometriosis is the most common cause of SCH bleeding (27/54). This is in agreement with the results detected by Sasaki, et al. [19] who found that the prev- 
alence of endometriosis and a younger age of patient at the time of hysterectomy are important risk factors for postoperative cervical stump bleeding.

As regard cervical stump neoplasia, the present study showed that the total number of stump cancer following SCH is not to be neglected. Our pathological reports revealed four cancer cases out of 132 symptomatizing patients, three invasive poorly differentiated squamous cell carcinomas (SCC) and one villoglandular adenocarcinoma (AC).

This is in agreement with Hellstrom, et al. [20] analysis of their results as the stump cancer cases had a worse stage pattern than the cancer cases in intact uterus. Earlier studies have found higher complication rates after stump cancer treatment (surgical and radiological), likely due to anatomical changes caused by subtotal hysterectomy [21,22].

Our four cancer patients were generally old with a median age 50 years. Three of them had diabetes. Their clinical symptoms included vaginal bleeding in non-menstruating female $(75 \%)$ and pelvic pain (25\%). No other symptoms were observed.

These results agree with Hellstrom, et al. [20] who stated that carcinoma of cervical stump is seen in $1-3 \%$ of patients with a history of SCH. Mostly among older women and had concurrent complicating conditions such as hypertension and diabetes [23]. On the other hand, Diaz-Feijoo, et al. [24] stated that since SCH are conducted infrequently, cervical stump cancer is a rare condition, accounting for just 2-5 percent of all cervical cancer cases worldwide. Nowadays, the trend has been towards preserving the cervix for more or less well-established reasons by performing SCH. In future may therefore expect to see an increase in the number of cervical stump cancers which necessitate regular cervical screening postoperatively for early detection of cancer [25].

Cervical intraepithelial neoplasia (CIN) is a premalignant pathological lesion. Despite the existing well-defined parameters, pathologists have a high rate of disagreement when it comes to histomorphologic diagnosis [26]. This can lead to both under- and over-reporting of clinically important cervical disease [27].

In the present study, all the inflammatory, neoplastic and the preneoplastic conditions of the studied cases (normal cervix and endometriosis were excluded) were further examined for p16 immuno-expression. It is considered as a good marker for identification of HPV infection in the cervix to confirm better diagnosis [28].

Several studies have reported p16 overexpression in cervical cancer as well as a high grade cervical intraepithelial neoplasia (CIN II-III) [29,30], but its clinical importance is still controversial. In our study, p16 immuno-staining of 53 studied cases showed $100 \%$ strong and diffuse block positivity: in all cancer cases. While all the chronic non-specific cervicitis and squamous metaplasia cases were negative. Regarding the cases of CIN, the specimens showed ambiguous immuno-staining expression; (strong focal / strong basal) in $50 \%, 63.2 \%$ and $80 \%$ cases of CIN I, CIN II, and CIN III, respectively.

P16 is a commonly used IHC marker; however, its interpretation is unique in the context of HPV-related lower anogenital lesions (Last project). Both quality and quantity of p16 immuno-reactivity affect its specificity in predicting high-risk HPV and high grade squamous intraepithelial lesion (CINII and CINIII) outcomes. It cannot be designated as positive or negative; rather, pathologists must consider multiple parameters such as staining intensity, extent, continuity, and location. When using p16 to evaluate CIN 2 lesions, pathologists need to be aware of the significance of an ambiguous $\mathrm{p} 16$ result [31].

Gonçalves, et al. [27] concluded that strong and diffuse p16 immuno-staining of cervical lesions caused by high risk HPV strains. Differently, lesions caused by low risk types displayed weak and focal p16 immuno-staining in the superficial and intermediate layers only.

It is worth noted that the accuracy of ambiguous p16 immuno-reactivity in predicting oncogenic HPV and HSIL outcome is significantly lower than that of the block-positive pattern but greater than negative staining [31].

As regard p16 immuno-staining in squamous metaplasia, our results were confirmed by Aslani, et al. [26] who observed that squamous metaplasia was entirely negative for $\mathrm{p} 16$.Our results are also more or less in agreement with Hebbar, et al. [7] who reported all the inflammatory samples showing no expression of p16/ INK4a and one case reported as atypical squamous metaplasia showed positive expression (20\%). They also found that All the invasive squamous cell carcinomas showed diffuse strong positive staining (100\%). While all the Adenocarcinoma cases showed diffuse strong positivity in all except one (83\%) which was a case of well differentiated adenocarcinoma.

Stoler, et al. [32] concluded that a positive p16 IHC result does not necessarily indicate the presence of a HSIL and the extent of diffuse p16 staining does not necessarily correlate with the grade of the lesion. As, CIN1 lesions may show one third, one half to two thirds, or even full thickness p16 staining within the squamous tissue.

Interestingly, only $30 \%$ of the ambiguous p16 immuno-stained lesions studied by Liu, et al. [15] were harboring HPV. While the remaining 70\% were negative for both low risk and high-risk HPV types. On the other hand, all the block positive p16 cervical specimens were harboring high-risk HPV strains and all negative p16 lesions were negative for them.

Moreover, Klaes, et al. [33] categorized p16 results into focal vs. diffuse. They detected HR-HPV in $27 \%$ of focal and $76 \%$ of diffuse cases. In contrast, block-positive p16 is strongly associated with the presence of HR-HPV [34]. These studies denote that p16 ambiguous with focal strong or focal basal expression in CIN cases based 
on H\&E that detected in our study may have a possibility of harboring high-risk HPV.

Data on the prevalence of HPV in Egypt's general population is currently unavailable. However, in north Africa, where Egypt is located, about 0.3 percent of women in the general population are reported to have cervical HPV-16/18 infection at any given time which is responsible for about $78.9 \%$ of invasive cervical cancers [35].

The strengths of the current study are its critical importance as it addresses several issues, the first is that SCH is linked to a lot of postoperative symptoms. Second issue is the adherence to postoperative follow up and pap smear in Egypt is lacking. The third issue is that small percentages of CIN, atypia and cancer cervix are detected in cervical stump following SCH. The fourth issue is the role of P16 immuno-staining in detection of cancer stump. The weakness of our study was the small sample size and its limitation to one region in Egypt.

\section{Conclusion}

P16 is important for screening of cervical stump cancer. It may be used as a complementary test to help distinguish between non-dysplastic and dysplastic lesions. While most p16 interpretation findings are clearly positive or negative, a few are ambiguous; they met some but not all the block positive pattern's requirements. CIN based on haematoxylin and eosin morphology which showed an ambiguous expression ; strong basal or strong focal staining should not be neglected as it may give a low possibility of high-risk HPV infection which need to be confirmed by further combination with HPV testing.

\section{Acknowledgement}

None.

\section{Conflicts of Interest}

Authors declare no conflict of interest.

\section{References}

1. Lethaby A, Mukhopadhyay A, Naik R (2012) Total versus subtotal hysterectomy for benign gynaecological conditions. Cochrane Database Syst Rev (4): CD004993.

2. Wilcox LS, Koonin LM, Pokras R, Strauss LT, Xia Z, et al. (1994) Hysterectomy in the United States, 1988-1990. Obstet Gynecol 83(4): 549-555.

3. Lieng M, Qvigstad E, Istre O, Langebrekke A, Ballard K (2008) Long-term outcomes following laparoscopic supracervical hysterectomy. BJOG 115(13): 1605-1610.

4. Okaro EO, Jones KD, Sutton C (2001) Long term outcome following laparoscopic supracervical hysterectomy. BJOG 108(10): 1017-1020.

5. Ghomi A, Hantes J, and Lotze EC (2005) Incidence of cyclical bleeding after laparoscopic supracervical hysterectomy. J Minim Invasive Gynecol 12(3): 201-205.

6. Lieng M, Lomo AB, Qvigstad E (2010) Long-term outcomes following laparoscopic and abdominal supracervical hysterectomies. Obstet Gynecol Int 2010: 989127.
7. Hebbar A, Murthy VS (2017) Role of p16/INK4a and Ki-67 as specific biomarkers for cervical intraepithelial neoplasia: An institutional study. J Lab Physicians 9(2): 104-110.

8. Malpica A, Matisic JP, Niekirk DV, Crum CP, Staerkel GA, et al. (2005) Kappa statistics to measure interrater and intrarater agreement for 1790 cervical biopsy specimens among twelve pathologists: qualitative histopathologic analysis and methodologic issues. Gynecol Oncol 99(3 Suppl 1): S38-S52.

9. Stoler MH, Schiffman M (2001) Interobserver reproducibility of cervical cytologic and histologic interpretations: realistic estimates from the ASCUS-LSIL Triage Study. Jama 285(11): 1500-1505.

10. Brown CA, Bogers J, Sahebali S, Depuydt CE, De Prins F, et al. (2012) Role of protein biomarkers in the detection of high-grade disease in cervical cancer screening programs. J Oncol 2012: 289315.

11. Darragh TM, Colgan TJ, Cox JT, Heller DS, Henry MR, et al. (2012) The Lower Anogenital Squamous Terminology Standardization Project for HPV-Associated Lesions: background and consensus recommendations from the College of American Pathologists and the American Society for Colposcopy and Cervical Pathology. J Low Genit Tract Dis 16(3): 205242.

12. Darragh TM (2015) The LAST Project and the diagnostic bottom line. Cytopathology 26(6): 343-345.

13. Hannoun- Lévi JM, Peiffert D, Hoffstetter S, Luporsi E, Bey P, et al. (1997) Carcinoma of the cervical stump: retrospective analysis of 77 cases. Radiother Oncol 43(2): 147-153.

14. Melnikow J, Nuovo J, Willan AR, Chan BK, Howell LP (1998) Natural history of cervical squamous intraepithelial lesions: a meta-analysis. Obstet Gynecol 92(4 Pt 2): 727-735.

15. Liu Y, Alqatari Mahfood, Sultan Kieran, Ye Fei, Gao Dana, et al. (2017) Using p16 immunohistochemistry to classify morphologic cervical intraepithelial neoplasia 2: correlation of ambiguous staining patterns with HPV subtypes and clinical outcome. Hum pathol 66: 144-151.

16. Dawood AS, Borg HM, and SA Atlam (2019) Assessment of the Egyptian gynecologists' clinical attitude and practice concerning route of hysterectomy. MOJ Womens Health 8(4): 255-259.

17.Zupi E, Zullo F, Marconi D, Sbracia M, Pellicano M, et al. (2003) Hysteroscopic endometrial resection versus laparoscopic supracervical hysterectomy for menorrhagia: a prospective randomized trial. Am J Obstet Gynecol 188(1): 7-12.

18. Shereen B Elbohoty, Ayman S Dawood, Adel E Elgergawy (2020) Gynecological Presentations Following Supravaginal Hysterectomy for Benign Pathologies. W J Gynecol Women's Health 3(2): 1-5.

19. Sasaki KJ, Cholkeri-Singh A, Sulo S, Miller CE (2014) Persistent bleeding after laparoscopic supracervical hysterectomy. JSLS 18(4).

20. Hellstrom AC, Hellman K, Pettersson BF, Andersson S (2011) Carcinoma of the cervical stump: fifty years of experience. Oncol Rep 25(6): 16511654.

21. Hellström AC, Sigurjonson T, Pettersson F (2001) Carcinoma of the cervical stump. The radiumhemmet series 1959-1987. Treatment and prognosis. Acta Obstet Gynecol Scand 80(2): 152-157.

22. Kottmeier HL (1964) Surgical and Radiation Treatment of Carcinoma of the Uterine Cervix. Experience by the Current Individualized Stockholm Technique. Acta Obstet Gynecol Scand 43(S2): 1-48.

23. Caruso S, Bruno MT, Cianci S, Di Pasqua S, Minona P, et al. (2019) Sexual Behavior of Women with Diagnosed HPV. J Sex Marital Ther 45(7): 569573.

24. Díaz-Feijoo B, Gil-Moreno A, Puig O, Martinez-Palones JM, Xercavins J (2005) Total laparoscopic radical trachelectomy with intraoperative sentinel node identification for early cervical stump cancer. J Minim Invasive Gynecol 12(6): 522-524. 
25. Shen Z, Zhou Y, Cheng Y, Li M, Wu D (2018) Retrospective analysis of surgery for cervical stump carcinoma at early stage. Mol Clin Oncol 8(2): 352-355.

26. Sari Aslani F, Safaei A, Pourjabali M, and Momtahan M (2013) Evaluation of Ki67, p16 and CK17 Markers in Differentiating Cervical Intraepithelial Neoplasia and Benign Lesions. Iran J Med Sci 38(1): 15-21.

27. Gonçalves Jessica ES, Andrade Cecilia Vianna de, Russomano Fabio B, Nuovo Gerard J, Amaro-Filho Sergio M, et al. (2017) The role of p16 as putative biomarker for cervical neoplasia: A controversial issue? Medical Express 4.

28. Thrall MJ (2016) Effect of Lower Anogenital Squamous Terminology Recommendations on the Use of p16 Immunohistochemistry and the Proportion of High-Grade Diagnoses in Cervical Biopsy Specimens. Am J Clin Pathol 145(4): 524-530.

29. Krishnappa P, Mohamad IB, Lin YJ, Barua A (2014) Expression of P16 in high-risk human papillomavirus related lesions of the uterine cervix in a government hospital, Malaysia. Diagn Pathol 9: 202.

30. Lin J, Albers AE, Qin J, Kaufmann AM (2014) Prognostic significance of overexpressed p16INK4a in patients with cervical cancer: a metaanalysis. PLoS One 9(9): e106384.
31. Babjuk M, Oosterlinck W, Sylvester R, Kaasinen E, Bohle A, et al. (2011) EAU guidelines on non-muscle-invasive urothelial carcinoma of the bladder, the 2011 update. Eur Urol 59(6): 997-1008.

32. Stoler MH, Wright TC, Jr, Ferenczy A, Ranger-Moore J, Fang Q et al. (2018) Routine Use of Adjunctive p16 Immunohistochemistry Improves Diagnostic Agreement of Cervical Biopsy Interpretation: Results from the CERTAIN Study. Am J Surg Pathol 42(8): 1001-1009.

33. Klaes R, Benner A, Friedrich T, Ridder R, Herrington S, et al. (2002) p16INK4a immunohistochemistry improves interobserver agreement in the diagnosis of cervical intraepithelial neoplasia. Am J Surg Pathol 26(11): 1389-1399.

34. Benevolo M, Mottolese M, Marandino F, Vocaturo G, Sindico R, et al. (2006) Immunohistochemical expression of $\mathrm{p} 16$ (INK4a) is predictive of HR-HPV infection in cervical low-grade lesions. Mod Pathol 19(3): 384391.

35. Bruni L Albero G, Serrano B, Mena M, Gómez D, Muñoz J, Bosch FX, de Sanjosé S (2019) Human Papillomavirus and Related Diseases in Egypt. ICO/IARC Information Centre on HPV and Cancer. 